\title{
Transfer Matrix Method for Dynamic Characteristics Analysis of Missile-Canister System in Silo
}

\author{
Anfeng Zhou ${ }^{1}$, Daokui $\mathrm{Li}^{1, *}$, Shiming Zhou ${ }^{1}$, Da Cui ${ }^{1}$, and Xuan Zhou ${ }^{1}$ \\ ${ }^{1}$ College of Aerospace Science and Engineering, National University of Defense Technology, Changsha, China
}

\begin{abstract}
In order to ensure the safety of the missile-canister system in silo during the earthquake, a modified transfer matrix method is provided to study the dynamic characteristics of the system. Firstly, a discrete viscoelastic connected double-beam model is developed taking account of the structural nonuniformity and the discrete distribution of the adapters. Secondly, the transfer matrix method of a single beam is modified to solve the problem of discrete connection between the two beams of the double-beam model. Then the natural circular frequencies and mode shapes are calculated by the proposed method, comparing with the finite element method (FEM). Finally, the influence of the stiffness of radial vibration isolators and adapters on the dynamic characteristics of the system is analysed. The comparison shows that the results of the proposed method are well consistent with the FEM calculations and the proposed method is validated. The variations of the first six natural circular frequencies with radial vibration isolator stiffness and adapter stiffness are obtained, which provides a basis for the seismic-relieving design.
\end{abstract}

\section{Introduction}

Once the missile-canister system erected in silo encounters an earthquake, it's easy to occur such accidents as shaking, collision with the silo body, local structural instability and internal equipment becoming ineffective caused by resonance. In order to ensure the safety and improve the seismic performance of the missile-canister system during the earthquake, it is necessary to analyse the transverse dynamic characteristics of the system.

At present, a launch vehicle or missile is always equivalent to a beam when analysing the vibration characteristics by the FEM and the transfer matrix method, which the shaking of liquid propulsion and the swinging of branch structure are well considered [1-2]. But for the complex missile-canister system, there are many simulation studies, while theoretical analysis is reported less. A simulation study on the flight dynamics of a vertical cold-launched missile during the launch phase was performed by Yan et al. [3], where the missile and canister were regarded as rigid bodies and the adapters were equivalent to spring damping elements. Similarly, lateral vibration characteristic of vertical launching for submarine-borne missile was investigated by Pei et al. [4] with the missile regarded as a rigid body and the seal ring simplified to an elastic solid, meanwhile, the effects of the rigidity and length of the seal ring on the lateral vibration were analysed and discussed. Furtherly, the coupling vibration of the missile-vehicle system under road excitation was studied by Wu et al. [5] with the energy method and FEM, in which the missile and vehicle were equivalent to a beam and a concentrated mass spring damping component respectively. As considering the structural flexibility, Zhang et al. [6] regarded the missile as an equivalent beam and the adapter and canister as elastic solids, then a dynamic simulation of the launching process of the cold-launched missile in silo was carried out. However, these studies are mainly focused on the multibody dynamic simulation and applied to attitude control of the missile during launch process, which cannot be used to investigate the seismic response and seismic-relieving design of the missile-canister system. Therefore, in this paper, the missile and the adapter can be regarded as a beam and a spring damping component respectively by referring to the modelling methods mentioned above. Furthermore, regarding the canister as a beam too, then the missile-canister system is equivalent to a special kind of double-beam system, which is favorable to theoretical analysis on the dynamic characteristics and optimization for seismic-relieving design.

As to the elastically connected double-beam system, numerous researches have been reported while the damping effect of the viscoelastic connected layer between the two beams is ignored [7-15]. Since the 21st century, the studies on the viscoelastic connected double-beam system increased gradually. The theoretical research of the double-beam system composed of two identical beams has been carried out by $\mathrm{Vu}$ et al. [16] and $\mathrm{Wu}$ et al. [17]. Alessandro et al. [18] proposed a Galerkin-type state-space approach which could be used to the inhomogeneous systems and any boundary conditions. $\mathrm{Li}$ et al. [19] analysed the dynamic characteristics of a double-beam system interconnected by a viscoelastic layer and with a Winkler layer below 
the system. After that, the influence of the mass effect of the viscoelastic layer on the double-beam system has been explored by Han et al. [20].

However, in all of the above researches, the elastic or viscoelastic connected double-beam systems have been regarded as continuous and uniform structures despite few of them concerned the nonuniformity of the beams. There are little reports about the discontinuous connected double-beam system. Wang et al. [21] studied the flexural vibration of a discretely connected Timoshenko double-beam and devoted to deal with the piecewise uniform beams, while the damping effect was ignored. Lee et al. [22] used the transfer matrix method to make vibration analysis of a partially elastic connected double-beam system with two uniform equivalent beams. Then Liu et al. [23] combined the distributed transfer function method and the Laplace transform method to solve the problem of partially viscoelastic connection and two diverse beams. But it isn't suitable for the system composed of the nonuniform beams.

In this paper, a double-beam model has been established which taking account of the nonuniformity and discreteness in actual structure and simplified reasonably, in order to accurately analyse the dynamic characteristics of the missile-canister system in silo and appropriately optimize the seismic-relieving design. And it is composed of multiple lumped masses, massless beams and several spring damping components. Then a transfer matrix method suitable for the double-beam model is proposed, so that the coupling behaviour between the two beams is solved conveniently and the natural circular frequencies and mode shapes are computed directly.

\section{Model and method}

\subsection{The double-beam model for missile-canister system}

A typical missile-canister system is shown in Fig. 1. For the greater risk of horizontal earthquake, the transverse vibration of the system has been the major research content in this paper. And a few assumptions are made as follows: (1) The missile and canister are both equivalent to a beam, ignoring the influences of surface deformation and gravity. (2) The adapter is very narrow relative to the length of the missile, so it's regarded as a spring damping oscillator without mass. (3) The radial vibration isolator between the canister and the silo is also a spring damping oscillator without mass. (4) The silo is regarded as a rigid body, which are able to move concomitantly with the surroundings during the earthquake.

Based on the above assumptions, the double-beam model exhibited in Fig. 2 has been founded to substitute the underground missile cold launch system. The beams $\mathrm{A}$ and $\mathrm{B}$ represent the canister and the missile respectively, which are both divided into $\mathrm{N}$ different segments according to the structural features. The radial vibration isolator is replaced by a spring $\mathrm{k} 1$ and $\mathrm{a}$ damper c1, meanwhile the $\mathrm{k} 2$ and c2 are substituted for the adapter.

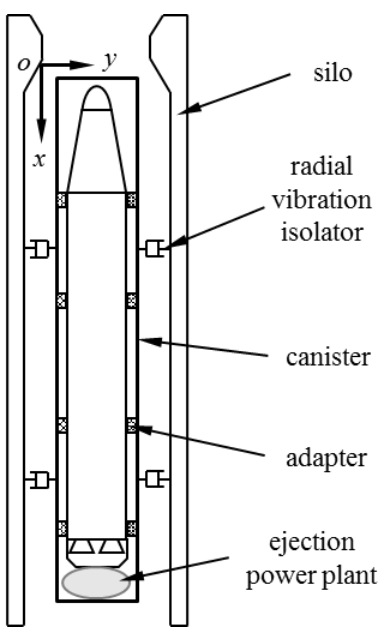

Fig. 1. Schematic of a missile-canister system

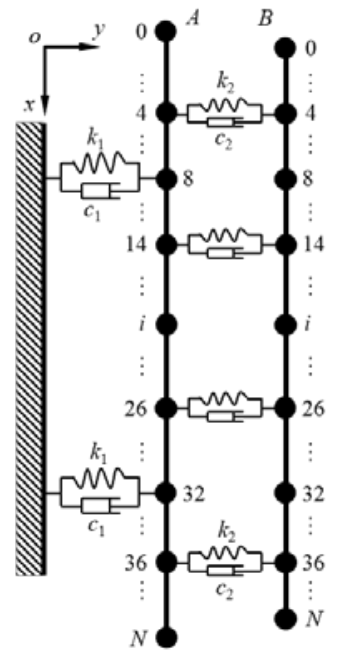

Fig. 2. The double-beam model of missile-canister system

\subsection{The transfer matrix method}

In this section, a transfer matrix technique is adopted to determine the natural circular frequencies and mode shapes of the double-beam model. As shown in Fig. 3, the $i$-th typical segment of the double-beam model is composed of the (i-1)-th group of nodes, the $i$-th group of nodes, the $i$-th group of massless beams and the $i$-th lumped masses.

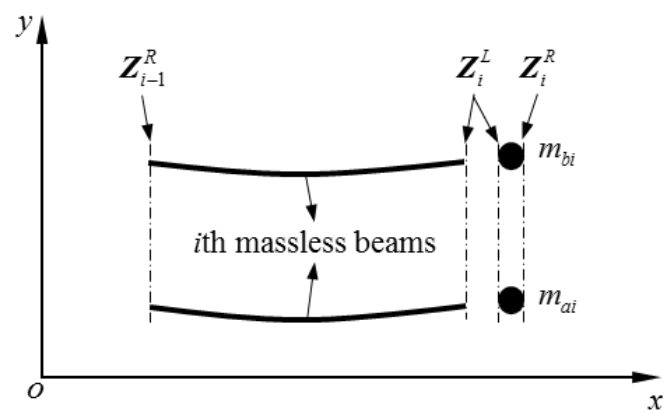

Fig. 3. The state of the $i$-th typical segment 
Taking account of the coupled effect between the two beams, the state vector of the $i$-th group of nodes is expressed as

$$
Z_{i}=\left[w_{a i}, \theta_{a i}, M_{a i}, F_{S a i}, w_{b i}, \theta_{b i}, M_{b i}, F_{S b i}\right]^{\mathrm{T}}
$$

where, $w_{a i}, \theta_{a i}, M_{a i}$, and $F_{S a i}$ represent the transverse deflection, rotation angle, bending moment and shear force in the section of the $i$-th node in beam A, and, $w_{b i}$, $\theta_{b i}, M_{b i}$, and $F_{S b i}$ represent the corresponding parameters in the section of the $i$-th node in beam B. Furtherly, the left and right side of the ith group of nodes are denoted as $\boldsymbol{Z}_{i}^{L}$ and $\boldsymbol{Z}_{i}^{R}$.

In the case of the double-beam model vibrating freely at its natural circular frequency $\omega$ with a zero damping, the following expressions are obtained from the equilibrium equations.

$$
\boldsymbol{Z}_{i}^{R}=\boldsymbol{P}_{i} \boldsymbol{Z}_{i}^{L}
$$

where $\boldsymbol{P}_{i}$ is the point transfer matrix, expressed as

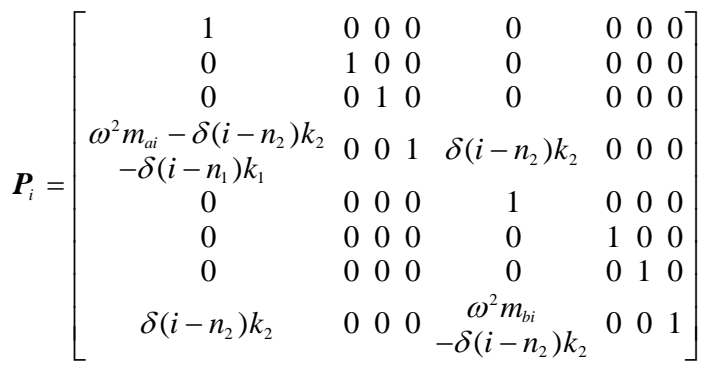

In Eq. 3, $m_{a i}$, and $m_{b i}$, represent the lumped mass of the $\mathrm{i}$-th node in beam $\mathrm{A}$ and beam $\mathrm{B}$ respectively, and a function $\delta$ is used to describe that whether the spring force acts on the lumped mass as below

$$
\delta(i-n)=\left\{\begin{array}{ll}
1 & i=n \\
0 & i \neq n
\end{array} \quad(i=0,1, \cdots, N)\right.
$$

Spesically, $n_{1}$ are the numbers of the lumped masses in beam A where the isolators connected, $n_{2}$ are the numbers of the lumped masses where the adapters connected.

For the $i$-th group of massless beams, the state relationship between the two ends is derived from the knowledge of material mechanics.

$$
\boldsymbol{Z}_{i}^{L}=\boldsymbol{F}_{i} \boldsymbol{Z}_{i-1}^{R}=\left[\begin{array}{cc}
\boldsymbol{F}_{a i} & \mathbf{0} \\
\mathbf{0} & \boldsymbol{F}_{b i}
\end{array}\right] \boldsymbol{Z}_{i-1}^{R}
$$

where $\boldsymbol{F}_{i}$ is the field transfer matrix. And the $\boldsymbol{F}_{a i}$ is denoted by

$$
\boldsymbol{F}_{a i}=\left[\begin{array}{cccc}
1 & l_{a i} & \frac{l_{a i}^{2}}{2 E_{a i} I_{a i}} & \frac{l_{a i}^{3}}{6 E_{a i} I_{a i}} \\
0 & 1 & \frac{l_{a i}}{E_{a i} I_{a i}} & \frac{l_{a i}^{2}}{2 E_{a i} I_{a i}} \\
0 & 0 & 1 & l_{a i}^{2} \\
0 & 0 & 0 & 1
\end{array}\right]
$$

In Eq. 6, $l_{a i}, E_{a i}$, and $I_{a i}$, are the length, Young's modulus and the cross-sectional moment of inertia of the $i$-th massless beam in beam A, while $\boldsymbol{F}_{b i}$ is obtained by replacing $a$ in the subscript of every element in $\boldsymbol{F}_{a i}$ with $b$.

Consequently, the state transfer relationship of the $i$ th typical segment is figured out by substituting Eq. 2 into Eq. 5.

$$
\boldsymbol{Z}_{i}^{R}=\boldsymbol{T}_{i} \boldsymbol{Z}_{i-1}^{R}
$$

where $\boldsymbol{T}_{i}=\boldsymbol{P}_{i} \boldsymbol{F}_{i}$ is the transfer matrix of the i-th segment.

In summary, the entire model is made of the 0th group of lumped masses and $N$ typical segments. As long as the point transfer matrix $\boldsymbol{P}_{0}$ and the transfer matrix of $N$ segments such as $\boldsymbol{T}_{1}, \boldsymbol{T}_{2}, \ldots, \boldsymbol{T}_{N}$ derived in turns, then $N+1$ transfer relationships such as $\boldsymbol{Z}_{0}^{R}=\boldsymbol{P}_{0} \boldsymbol{Z}_{0}^{L}$, $\boldsymbol{Z}_{1}^{R}=\boldsymbol{T}_{1} \boldsymbol{Z}_{0}^{R}, \boldsymbol{Z}_{2}^{R}=\boldsymbol{T}_{2} \mathbf{Z}_{1}^{R}, \ldots, \boldsymbol{Z}_{N}^{R}=\boldsymbol{T}_{N} \boldsymbol{Z}_{N-1}^{R}$ can be obtained. Furthermore, substituting $\boldsymbol{Z}_{0}, \boldsymbol{Z}_{1}, \ldots, \boldsymbol{Z}_{N-1}$ into the next relationship successively, the total transfer relationship between the state vectors of both ends of the doublebeam model is determined as follow

$$
\mathbf{Z}_{N}^{R}=\mathbf{T Z}_{0}^{L}
$$

where $\boldsymbol{T}=\boldsymbol{T}_{N} \boldsymbol{T}_{N-1} \cdots \boldsymbol{T}_{1} \boldsymbol{P}_{0}$ is called the assembled transfer matrix.

According to the preceding derivation, each element in the assembled transfer matrix is a function of $\omega$. Therefore, the Eq. 8 is also represented as

$$
\boldsymbol{Z}_{N}^{R}=\left[\begin{array}{cccc}
t_{11}(\omega) & t_{12}(\omega) & \cdots & t_{18}(\omega) \\
t_{21}(\omega) & t_{22}(\omega) & \cdots & t_{28}(\omega) \\
\vdots & \vdots & \ddots & \vdots \\
t_{81}(\omega) & t_{82}(\omega) & \cdots & t_{88}(\omega)
\end{array}\right] \boldsymbol{Z}_{0}^{L}
$$

Considering the real engineering practices, the boundary conditions of the double-beam model is given as follows

$$
\left\{\begin{array}{l}
\mathbf{Z}_{0}^{L}=\left[w_{a 0}^{L}, \theta_{a 0}^{L}, 0,0, w_{b 0}^{L}, \theta_{b 0}^{L}, 0,0\right]^{\mathrm{T}} \\
\mathbf{Z}_{N}^{R}=\left[w_{a N}^{R}, \theta_{a N}^{R}, 0,0, w_{a N}^{R}, \theta_{a N}^{R}, 0,0\right]^{\mathrm{T}}
\end{array}\right.
$$

Substituting Eq. 10 into Eq. 9, gives a set of homogeneous linear equations whose coefficients are various functions of $\omega$.

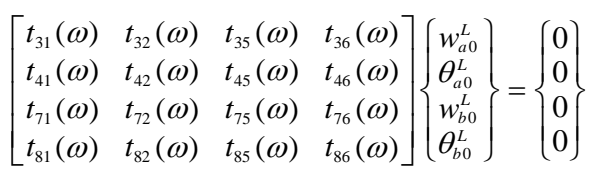

Obviously, there are nonzero solutions for Eq. 11 once the vibration is present, so the coefficients of Eq. 11 must satisfy the following formula

$$
\operatorname{det}\left[\begin{array}{cccc}
t_{31}(\omega) & t_{32}(\omega) & t_{35}(\omega) & t_{36}(\omega) \\
t_{41}(\omega) & t_{42}(\omega) & t_{45}(\omega) & t_{46}(\omega) \\
t_{71}(\omega) & t_{72}(\omega) & t_{75}(\omega) & t_{76}(\omega) \\
t_{81}(\omega) & t_{82}(\omega) & t_{85}(\omega) & t_{86}(\omega)
\end{array}\right]=0
$$

As a result, the natural circular frequencies are determined by Eq. 12 . While substituting $\omega$ back into Eq. 
11, the relationship of $\theta_{a 0}^{L} / w_{a 0}^{L}, w_{b 0}^{L} / w_{a 0}^{L}$ and $\theta_{b 0}^{L} / w_{a 0}^{L}$ are figured out. Then the corresponding state vector $\boldsymbol{Z}_{0}^{L}$ can be obtained if the $w_{a 0}^{L}$ set as 1 according to the normalized mode. Thus $\boldsymbol{Z}_{0}^{R}$ is determined by substituting $\boldsymbol{Z}_{0}^{L}$ into Eq. 2, and $\boldsymbol{Z}_{1}^{R}, \boldsymbol{Z}_{2}^{R}, \ldots, \boldsymbol{Z}_{N-1}^{R}$ are obtained by substituting $\boldsymbol{Z}_{0}^{R}$ back into the $N+1$ transfer relationships metioned above. Moreover, a new column vector $\phi_{w}$, i.e. the mode-shape vector of the present $\omega$ can be obtained by extracting $\left(w_{a 0}^{L}, w_{b 0}^{L}\right),\left(w_{a 1}^{L}, w_{b 1}^{L}\right), \ldots,\left(w_{a N}^{L}, w_{b N}^{L}\right)$ from $\boldsymbol{Z}_{1}^{R}, \boldsymbol{Z}_{2}^{R}, \ldots, \boldsymbol{Z}_{N}^{R}$, which can be expressed as $\phi_{w}=\left[w_{a 0}^{R}, w_{a 1}^{R}, \cdots, w_{a N}^{R}, w_{b 0}^{R}, w_{b 1}^{R}, \cdots, w_{b N}^{R}\right]^{\mathrm{T}}$. Finally, the modeshape matrix is determined as $\boldsymbol{\Phi}_{w}=\left[\phi_{w}^{1}, \phi_{w}^{2}, \cdots, \phi_{w}^{j}, \cdots\right]$ where $\phi_{w}^{j}$ represent the mode-shape vector of $j$-th mode.

In a similar way, the mode-shape matrix of rotation angle $\boldsymbol{\Phi}_{\theta}$, the mode-shape matrix of bending moment $\boldsymbol{\Phi}_{M}$ and the mode-shape matrix of shear force $\boldsymbol{\Phi}_{F}$ can be obtained by extracting $\theta^{R}, M^{R}$ and $F_{S}^{R}$ respectively from all the state vectors.

\section{Numerical study}

In order to verify the correctness of the method proposed in this paper, a numerical example is given and the results are compared with the FEM calculations. The basic parameters are as follows

$N=40, n_{1}=(8,32), n_{2}=(4,14,26,36), k_{1}=3.5 \times 10^{5} \mathrm{~N} / \mathrm{m}$, $k_{2}=5.5 \times 10^{5} \mathrm{~N} / \mathrm{m}, c_{1}=1000 \mathrm{Ns} / \mathrm{m}, c_{2}=200 \mathrm{Ns} / \mathrm{m}$.

Additionally, the model parameters of beam A and B are shown in Tab. 1.

Table 1. Model parameters of beam A and B.

\begin{tabular}{|c|c|}
\hline Beam A & Beam B \\
\hline$E_{a 1} \sim E_{a 40}=7 \times 10^{10} \mathrm{~Pa}$ & $E_{a 1} \sim E_{a 40}=10 \times 10^{10} \mathrm{~Pa}$ \\
\hline$I_{a 1}=0.8250 \mathrm{~m}$ & \\
$I_{a 2} \sim l_{a 8}=0.6250 \mathrm{~m}$ & $I_{b 1} \sim l_{b 8}=0.6250 \mathrm{~m}$ \\
$I_{a 9} \sim l_{a 32}=0.4617 \mathrm{~m}$ & $I_{b 9} \sim l_{b 32}=0.4617 \mathrm{~m}$ \\
$l_{a 33} \sim l_{a 39}=0.6250 \mathrm{~m}$ & $I_{b 33} \sim l_{b 40}=0.6250 \mathrm{~m}$ \\
$l_{a 40}=0.9250$ & $I_{b 1} \sim I_{b 8}=6.032 \times 10^{-4} \mathrm{~m}^{-3}$ \\
& $I_{b 9} \sim I_{b 20}=1.568 \times 10^{-3} \mathrm{~m}^{-3}$ \\
$I_{a 1} \sim I_{a 40}=3.217 \times 10^{-3} \mathrm{~m}^{-3}$ & $I_{b 21} \sim I_{b 32}=2.036 \times 10^{-3} \mathrm{~m}^{-3}$ \\
& $I_{b 32} \sim I_{b 40}=1.325 \times 10^{-3} \mathrm{~m}^{-3}$ \\
\hline$m_{a 0}=24.88 \mathrm{~kg}$ & $m_{b 0}=39.93 \mathrm{~kg}$ \\
$m_{a 1}=43.73 \mathrm{~kg}$ & $m_{b 1} \sim m_{b 7}=67.86 \mathrm{~kg}$ \\
$m_{a 2} \sim m_{a 7}=37.70 \mathrm{~kg}$ & $m_{b 8}=80.58 \mathrm{~kg}$ \\
$m_{a 8}=31.42 \mathrm{~kg}$ & $m_{b 9} \sim m_{b 19}=93.31 \mathrm{~kg}$ \\
$m_{a 9} \sim m_{a 31}=25.13 \mathrm{~kg}$ & $m_{b 20}=97.55 \mathrm{~kg}$ \\
$m_{a 32}=31.42 \mathrm{~kg}$ & $m_{b 21} \sim m_{b 31}=101.79 \mathrm{~kg}$ \\
$m_{a 33} \sim m_{a 38}=37.70 \mathrm{~kg}$ & $m_{b 32}=95.00 \mathrm{~kg}$ \\
$m_{a 39}=46.75 \mathrm{~kg}$ & $m_{b 33} \sim m_{b 33}=88.22 \mathrm{~kg}$ \\
$m_{a 40}=27.90 \mathrm{~kg}$ & $m_{b 40}=44.11 \mathrm{~kg}$ \\
\hline
\end{tabular}

The first six natural circular frequencies and mode shapes of the double-beam model are summarized in Tab. 1 and Fig. 4. It can be seen from Tab.2 that the relative errors of the first six natural circular frequencies between the transfer matrix method presented in this paper and the FEM are within 2.0\%. Especially, the relative errors of the first four modes are less than $0.2 \%$.
Table 2. Natural circular frequencies of double-beam model.

\begin{tabular}{|c|c|c|c|}
\hline Mode & $\begin{array}{c}\omega(\mathbf{r a d} / \mathbf{s}) \\
\text { FEM }\end{array}$ & $\begin{array}{c}\omega(\mathbf{r a d} / \mathbf{s}) \\
\text { Present }\end{array}$ & $\begin{array}{c}\text { relative } \\
\text { error }\end{array}$ \\
\hline 1 & 9.9503 & 9.9649 & $0.15 \%$ \\
\hline 2 & 11.2720 & 11.2870 & $0.13 \%$ \\
\hline 3 & 49.8190 & 49.8999 & $0.16 \%$ \\
\hline 4 & 53.0842 & 53.1844 & $0.19 \%$ \\
\hline 5 & 57.7172 & 58.3147 & $1.04 \%$ \\
\hline 6 & 107.2358 & 109.2737 & $1.90 \%$ \\
\hline
\end{tabular}

Furthermore, the mode shapes determined by the presented method are in excellent agreement with the FEM results as shown in Fig. 4, where the calculations are normalized based on the FEM for comparison. Therefore, the accuracy and validity of the presented method are confirmed.

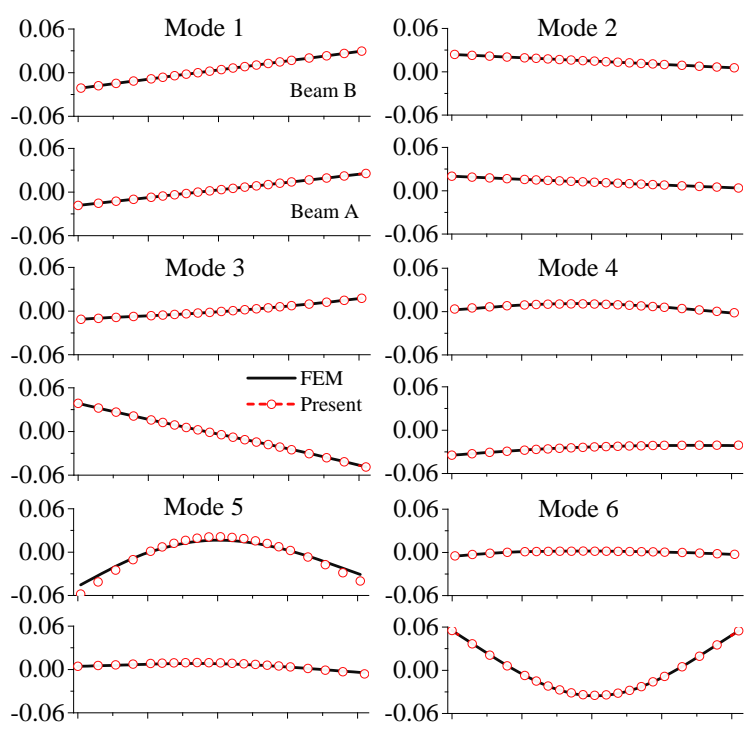

Fig. 4. The first six mode shapes of double-beam model

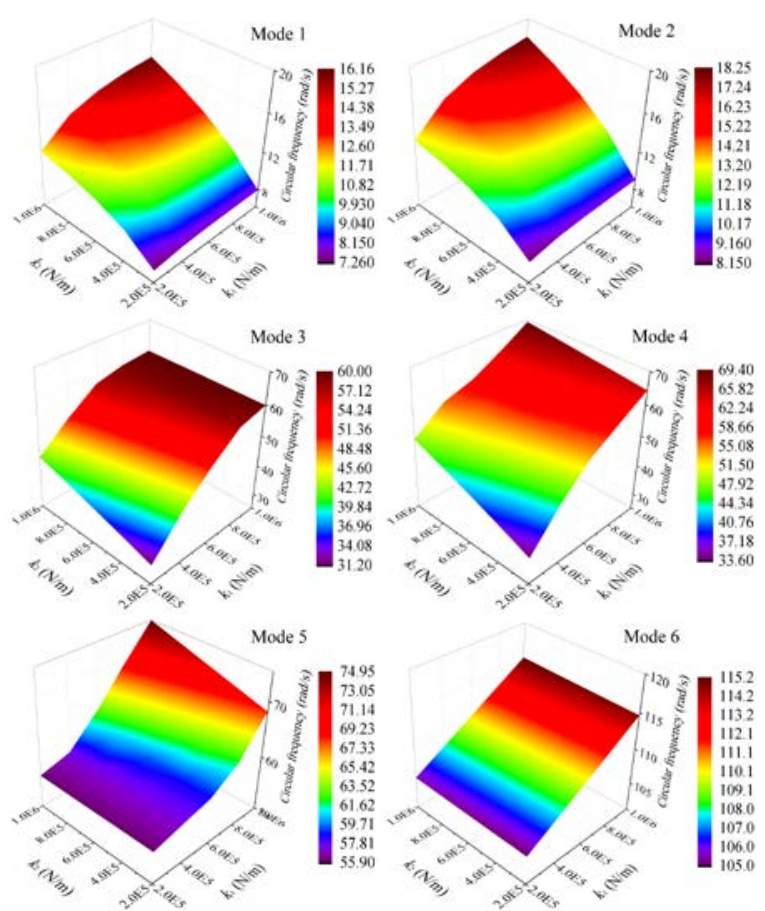

Fig. 5. Natural circular frequencies versus $k_{1}$ and $k_{2}$ 
The variations of the first six natural frequencies of the double-beam model with $k_{1}$ and $k_{2}$ are shown in Fig. 5. Generally the first six natural frequencies are increased as the radical vibration isolator stiffness and the adapter stiffness raised. Moreover, the $1^{\text {st }}$ and $2^{\text {nd }}$ order natural frequencies are primarily affected by $k_{2}$, while the $3^{\text {rd }}$ to $6^{\text {th }}$ order natural frequencies are more sensitive to $k_{1}$. Particularlly the 6th natural frequency is hardly varied with $k_{2}$ and represents a linear correlation with $k_{1}$. These results provide a basis for the seismicrelieving design in some degree.

\section{Conclusions}

The study on the dynamic characteristic of underground missile cold launch system is carried out in this paper, showing the following conclusions:

A double-beam model is established for underground missile cold launch missile system, taking account of the structural nonuniformity and the discrete distribution of the adapters seriously. And the model is divided into several lumped masses and massless beams to form a chain-like structure, so that the natural circular frequencies and mode shapes of the model can be determined directly by the transfer matrix technique. Additionally, the proposed method is simple and feasible in engineering application and quite convenient for computer programming.

The numerical study indicates that the results of the proposed method are well consistent with the FEM calculations. Meanwhile the accuracy and efficiency of the double-beam model are confirmed sufficiently in the application of proposed method for nonuniform doublebeam structure interconnected with discrete viscoelastic components.

The first six natural frequencies of the double-beam model are increased as the radical vibration isolator stiffness and the adapter stiffness raised. The 1st and 2nd order natural frequencies are primarily affected by k2, while the 3rd to 6th order natural frequencies are more sensitive to $\mathrm{k} 1$, which provide a basis for the seismicrelieving design.

Moreover, the double-beam model proposed in this paper can be applied to analyse the dynamic response and optimize the seismic-relieving design of the system furtherly, which has great advantage of achieving the whole process of the research from modelling, analysis to design.

\section{References}

1. Y. Hao, G. Tang, D.Y. Xu Q.L. Yang. AIAA J., 53, 3297-3304 (2015)

2. K.A. Alev, E. Seher, O.K. Metin. Int. J. Mech. Eng. Robot. Res., 8, 477-482 (2019)

3. P.Y. Yan, G.Z. Liang, Y.Z. Lu, Z.H. Qi. 3rd International Conference on Mechanical Engineering and Automation Science (ICMEAS), (Birmingham, England, 2017)
4. X. Pei , Y.W. Zhang, X.L. Yuan, J.H. Zhang. Acta Armamentarii, 30, 1056-1060 (2009)

5. S. Wu, H. Ai, Y. Guo. Journal of Southeast University (Natural Science Edition), 43, 1055-1061 (2013) (in Chinese)

6. X. Zhang, B.L. Liu, X. Zheng, P.C. Deng, G.J. He. Missiles and Space Vehicles, 362, 85-90 (2018) (in Chinese)

7. J.M. Seelig, W.H. Hoppmann. J. Acoust. Soc. Am., 36(1): 93-99 (1964)

8. P.G. Kessel. J. Acoust. Soc. Am., 40, 684-687 (1966)

9. T.R. Hamada, H. Nakayama, K. Hayashi. Bulletin of JSME, 26, 1936-1942 (1983)

10. Q.J. Hao, W.J. Zhai, Z.B. Chen. Arch. Appl. Mech., 88, 741-754 (2018)

11. J. Li, H.X. Hua. Finite Elem. Anal. Des., 43, 11551168 (2007)

12. S. Vladimir, K. Predrag. Int. J. Mech. Sci., 60, 59-71. (2012)

13. K. Predrag, P. Ratko, K. Danilo. Mech. Res. Commun., 56, 83-89 (2014)

14. Q.B. Mao, N. Wattanasakulpong. Int. J. Mech. Sci., 93, 1-7 (2014)

15. O.O. Agboola, J.A. Gbadeyan, S.A. Iyase. World Congress on Engineering (London, U.K., 2017)

16. H.V. Vu, A.M. Ordonez, B.H. Karnopp. J. Sound Vib., 229(4): 807-822. (2000)

17. Y.X. Wu, Y.F. Gao. J. Eng. Mech., doi: 10.1061/ASCE)EM.1943-7889.0000900 (2005)

18. P. Alessandro, A. Sondipon. J. Sound Vib., 330, 6372-6386 (2011)

19. Y.X. Li, Z.J. Hu, L.Z. Sun. Int. J. Mech. Sci., 105: 291-303 (2016)

20. F. Han, D.H. Dan, W. Cheng. Compos. Struct., 193, 295-305 (2018)

21. J. Wang, Z.G. Zhang, H.X. Hua. Int. J. Appl. Mech., doi: 10.1142/S1758825116500678. (2016)

22. J. Lee, S. Wang. Int. J. Appl. Mech., doi: 10.1142/S1758825117500934 (2017)

23. S.B. Liu, B.G Yang. Compos. Struct., 212, 598-608 (2019) 